\title{
The impact of education on risk factors and the occurrence of multimorbidity in the EPIC-Heidelberg cohort
}

\author{
Gabriele Nagel*1, Richard Peter ${ }^{1}$, Stefanie Braig ${ }^{1}$, Silke Hermann², \\ Sabine Rohrmann ${ }^{2}$ and Jakob Linseisen ${ }^{2}$
}

\begin{abstract}
Address: ${ }^{1}$ Institute of Epidemiology, Ulm University, Ulm, Germany and 2Division of Cancer Epidemiology, German Cancer Research Centre Heidelberg, Germany

Email: Gabriele Nagel* - gabriele.nagel@uni-ulm.de; Richard Peter - richard.peter@uni-ulm.de; Stefanie Braig - stefanie.braig@uni-ulm.de; Silke Hermann - s.hermann@dkfz.de; Sabine Rohrmann - s.rohrmann@dkfz.de; Jakob Linseisen - j.linseisen@dkfz.de

* Corresponding author
\end{abstract}

Published: II November 2008

BMC Public Health 2008, 8:384 doi: I0.1 I86/I47I-2458-8-384

This article is available from: http://www.biomedcentral.com/ I47/-2458/8/384

(C) 2008 Nagel et al; licensee BioMed Central Ltd.

This is an Open Access article distributed under the terms of the Creative Commons Attribution License (http://creativecommons.org/licenses/by/2.0), which permits unrestricted use, distribution, and reproduction in any medium, provided the original work is properly cited.
Received: 4 June 2008

Accepted: II November 2008

\begin{abstract}
Background: In aging populations, the prevalence of multimorbidity is high, and the role of socioeconomic status and its correlates is not well described. Thus, we investigated the association between educational attainment and multimorbidity in a prospective cohort study, taking also into account intermediate factors that could explain such associations.
\end{abstract}

Methods: We included 13,78I participants of the Heidelberg cohort of the European Prospective Investigation into Cancer and Nutrition (EPIC), who were 50-75 years at the end of follow-up. Information on diet and lifestyle was collected at recruitment (1994-1998). During a median follow-up of 8.7 years, information on chronic conditions and death were collected.

Results: Overall, the prevalence of multimorbidity ( $>=2$ concurrent chronic diseases) was $67.3 \%$. Compared to the highest educational category, the lowest was statistically significantly associated with increased odds of multimorbidity in men $(\mathrm{OR}=\mathrm{I} .43 ; 95 \% \mathrm{Cl} \mathrm{I} .28-\mathrm{I} .6 \mathrm{I})$ and women $(\mathrm{OR}=$ I.33; $95 \% \mathrm{Cl}$ I.I8-I.57). After adjustment, the positive associations were attenuated (men: $\mathrm{OR}=$ I.28; $95 \%$ Cl I.12-I.46; women: OR = I.16; $95 \%$ Cl 0.99-I.36). Increasing BMI was more strongly than smoking status an intermediate factor in the association between education and multimorbidity.

Conclusion: In this German population, the prevalence of multimorbidity is high and is significantly associated with educational level. Increasing BMI is the most important predictor of this association. However, even the fully adjusted model, i.e. considering also other known risk factors for chronic diseases, could not entirely explain socio-economic inequalities in multimorbidity. Educational level should be considered in the development and implementation of prevention strategies of multimorbidity.

\section{Background}

Aging populations are characterized by the co-occurrence of multiple chronic conditions. Two or more concurrent chronic conditions are defined as multimorbidity, whereas comorbidity refers to an index disease $[1,2]$. In the Netherlands, the prevalence of multimorbidity was 
$33.6 \%$ in 50-59 year old men, $60.5 \%$ in $60-79$ year old men, and $74.2 \%$ in men 80 years or older, among women the prevalence was $35.9 \%, 64.9 \%$, and $79.9 \%$, respectively [2]. Similar numbers were reported from Canada and the U.S. [3,4]. Most studies on multimorbidity were performed in general practice setting [2] or on aggregated population level $[5,6]$.

Socio-economic inequalities were shown to be associated with morbidity and mortality [5-11]. Educational attainment, occupational social class, and income are established indicators of socio-economic position (SEP) [12]. Moreover, pathways through which the socio-economic position influences morbidity have been proposed [13]. For example, SEP differences are likely to reflect differences in smoking [14], diet [15], alcohol consumption and physical activity [16]. Living conditions, chronic stress and access to the health care system were also examined in relation to health disparities [5]. Education as a marker for SEP may be associated with the prevalence of chronic medical conditions in later life. Because multimorbidity has an impact on quality of life and on life expectancy at the individual and population level, the knowledge about modifiable risk factors is valuable $[2,17]$. Up to now little is known about the determinants and the effect of potential risk factors such as diet and lifestyle on the prevalence of multimorbidity in the general population [17].

Thus, the aims of this study were to investigate the association between educational attainment and multimorbidity and to examine the mediating role of health behaviour among participants aged 50 years or older in the EPICsubcohort of Heidelberg.

\section{Methods \\ Study Subjects}

Between June 1994 and October 1998, 24,540 participants were recruited in the EPIC-Heidelberg cohort, contributing to the European Prospective Investigation into Cancer and Nutrition (EPIC) [18]. Subjects were recruited from the general population [19]. For the present analysis participants younger than 50 years at the end of follow-up $(\mathrm{N}=10,759)$ were excluded. Thus, the analytic cohort comprised 13,781 participants aged between 50 and 75 years at the end of a mean follow-up of 8.7 years.

The baseline assessment included a self-administered food frequency questionnaire (FFQ) and standardized anthropometric measurements including height and weight. Nutrient intake data were calculated from the food consumption data by means of the German Food Composition Tables. The participants were also asked to provide detailed information about their smoking history, physical activity and medical history. The investiga- tion was approved by the ethics committee at the Heidelberg University.

\section{Follow-up and outcome}

Follow-up of the cohort began at the date of recruitment and ended at either death or date of the last contact, whichever came first. Prevalent chronic diseases at recruitment were assessed during face-to-face interview. Incident cases of cancer and other chronic diseases were identified by active follow-up. The follow-up consisted of a questionnaire on physician diagnosed chronic conditions and the year of diagnosis. Chronic diseases were regrouped into cancer (all except non-melanoma skin cancer), diabetes mellitus, thyroid disease, stroke, myocardial infarction (MI), heart diseases (except MI), lung diseases (asthma, chronic obstructive pulmonary disease), gastrointestinal diseases (reflux esophagitis, chronic gastritis, gastric ulcer, Crohn's disease, ulcerative colitis, chronic diverticulitis), liver/kidney diseases, pancreatitis, muscle/bone diseases (rheumatic disease, osteoporosis), diseases of the central nervous system (multiple sclerosis, Alzheimer disease, Parkinson's disease), hypertension, dyslipidemia, and hyperuricemia. All self-reports on incident cases of cancer were verified by comparison with the medical records. However, medical verification of other chronic diseases has not been done, except for a few small validation/pilot studies on incident cases of myocardial infarction, stroke, asthma [20], and diabetes. During follow-up, 800 decedents (576 men and 224 women) were registered, confirmed by death certificates, and all-cause mortality was determined. Participation rate was over 90\% in each follow-up round. At the end of the $3^{\text {rd }}$ follow-up, $5.8 \%$ of the study participants were lost for follow-up.

The number of concurrent chronic conditions was counted to compute a score of morbidity $(0$ to $\geq 6)$. Multimorbidity (yes/no) was defined as two or more concurrent chronic conditions. In addition, multiple metabolic diseases is defined as the concomitant occurrence of metabolic diseases $(0,1,2, \geq 3)$ including diabetes, hypertension, dyslipidemia and hyperuricemia; the dichotomous variable 'metabolic diseases' (yes/no) reflects the existence of any metabolic disease.

\section{Predictors}

Educational attainment was used to characterise a participant's SEP. The highest education achieved was measured by questionnaire at recruitment and summarized according to the German schooling system with three degrees: primary school or less (low), secondary school (middle), and grammar school (high). Subjects without school graduation ( $\mathrm{N}=113$ ) were assigned to the lowest educational level. The highest category of educational attainment was used as the reference group. 
As covariates known or potential risk factors of multimorbidity were considered. Smoking status was categorized as never smoker, ex-smoker or current smoker. Body mass index (BMI) was calculated as weight $(\mathrm{kg}) /$ height $^{2}\left(\mathrm{~m}^{2}\right)$. Alcohol consumption (g/day), fruit and vegetable intake (g/day) were included in the models as continuous variables. Physical activity was classified as inactive, moderately inactive, moderately active and active based on occupational activity, cycling, and sports [21].

\section{Statistical analysis}

This is a cross-sectional analysis of multi-morbidity, using all available information on prevalent chronic diseases collected at recruitment of the cohort and during followup rounds. Information on lifestyle factors, diet, and anthropometric data (measurements) was collected at recruitment. The prevalence of chronic conditions was analyzed in relation to educational attainment stratified by sex, because the association between educational level and health outcome could vary by sex as former reports have shown $[2,3,22]$. Age-adjusted logistic regression models were calculated to examine predictors of multimorbidity and Cox proportional hazard models (HR) for mortality, respectively. In order to explore mediating effects we introduced smoking status and increasing BMI (per increments of $5 \mathrm{~kg} / \mathrm{m}^{2}$ ) in the age-adjusted models. Adjusted odds ratios $\left(\mathrm{OR}_{\mathrm{adj}}\right)$ and $95 \%$ confidence intervals $(95 \% \mathrm{CI})$ for morbidity were calculated by inclusion of age (continuous, year), BMI (continuous, $\mathrm{kg} / \mathrm{m}^{2}$ ), fruit intake (continuous, g/day), vegetable intake (continuous, g/day), alcohol consumption (continuous, g/day), smoking habits, and total physical activity in the models. Whether the associations differed by sex or age was assessed by including a cross-product term in the logistic regression equitation, and $\mathrm{p}$ for interaction was calculated with the log-likelihood test. For evaluation of the impact of the single multimorbidity scores, multinomial logistic regression models applying the generalized logit function (glogit) in PROC LOGISTIC were calculated. SAS statistical software 9.13 (SAS institute Inc, Cary, NC) was used for all calculations. A p-value $<0.05$ was used to determine statistical significance.

\section{Results}

Among 13,781 participants aged 50 to 75 years, the prevalence of multimorbidity was $67.3 \%$ in men and $67.4 \%$ in women. In men and women, dyslipidemia, hypertension, and gastro-intestinal diseases were the most common chronic conditions. High educational level was related to lower prevalence of dyslipidemia, hypertension, insulindependent diabetes, hyperuricemia, myocardial infarction, stroke and cancer in both men and women (Table 1). The distribution of baseline characteristics stratified by educational level and sex are displayed in table 2. Subjects in the highest education category were leaner, whereas in women no educational gradient was found. Irrespective of educational level men were prone to a more sedentary lifestyle.

The associations between education and morbidity by sex are shown in table 3 . Low education category shows strong gradients with increasing number of chronic diseases overall as well as for metabolic diseases. All relationships were statistically significant, except for metabolic diseases in men.

In the adjusted models, low educational attainment of men was statistically significantly associated with an increased prevalence of most chronic diseases except for cancer and stroke in men (data not shown). Among women no associations were found for cancer, myocardial infarction, pancreatic disease, liver/kidney diseases and thyroid disease. Diseases of the heart (other than MI), lung, liver/kidney, and central nervous system were not associated with educational level in men and women (data not shown).

Table 4 displays the age- and multivariate adjusted odds ratios for multimorbidity by education and sex. In the ageadjusted models, the associations between educational level and multimorbidity were slightly stronger in men (middle vs. high level OR $=1.32 ; 95 \%$ CI $1.12-1.55$ and low vs. high level OR $=1.43 ; 95 \%$ CI $1.28-1.61$ ) than in women (middle vs. high level OR $=1.13 ; 95 \%$ CI 0.97 1.33 and low vs. high level OR $=1.33 ; 95 \%$ CI 1.18-1.57). In women, the educational level had stronger impact on the occurrence of metabolic diseases (low vs. high level $\mathrm{OR}=1.64 ; 95 \%$ CI 1.42-1.90) than in men (low vs. high level OR $=1.38 ; 95 \%$ CI 1.22-1.57). However, none of these suggested statistical interactions between sex and education were of statistical significance (multimorbidity $\mathrm{p}=0.670$ and metabolic disease $\mathrm{p}=0.447)$. Differences in the strength of the association may be predominantly attributed to diabetes, which was more strongly associated with education in women than in men. By adjustment for potential risk factors the associations for multimorbidity, metabolic disease and mortality were attenuated. Concerning all-cause mortality, the introduction of smoking status and BMI in age-adjusted models attenuated the estimates with higher mortality among subjects in the lowest educational category.

The introduction of BMI, but not smoking, in the ageadjusted models attenuated the estimates for multimorbidity and metabolic disease indicating that increasing BMI may contribute as intermediate factor to the relationship with education. Smoking habits had a moderate effect on the association between education and mortality in men, while increasing BMI had little effect on this relationship. In women, smoking status and increasing BMI 
Table I: Prevalence of chronic conditions among participants aged 50 to 75 years in the in the EPIC-HD cohort by educational level and sex

\begin{tabular}{|c|c|c|c|c|c|c|c|c|}
\hline \multirow{2}{*}{$\begin{array}{l}\text { Educational level } \\
\text { Men }\end{array}$} & \multicolumn{2}{|c|}{ Low } & \multicolumn{2}{|c|}{ Middle } & \multicolumn{2}{|c|}{ High } & \multicolumn{2}{|c|}{ Total } \\
\hline & $N$ & $\%$ & $N$ & $\%$ & $N$ & $\%$ & $N$ & $\%$ \\
\hline Dyslipidemia & 2237 & 58.8 & 614 & 57.7 & 1262 & 52.2 & 4113 & 56.5 \\
\hline Hyperuricemia & 708 & 19.0 & 140 & 13.3 & 275 & 11.5 & 1123 & 15.7 \\
\hline Diabetes mellitus all & 643 & 16.9 & 129 & 12.1 & 241 & 10.0 & 1013 & 13.9 \\
\hline insulin dependent & 131 & 3.4 & 23 & 2.2 & 50 & 2.1 & 204 & 2.8 \\
\hline Thyroid disease & 271 & 7.2 & 91 & 8.7 & 183 & 7.6 & 545 & 7.6 \\
\hline Hypertension & 2175 & 57.1 & 594 & 55.8 & 1188 & 49.2 & 3957 & 54.3 \\
\hline Stroke & 204 & 5.4 & 41 & 3.9 & 78 & 3.2 & 323 & 4.4 \\
\hline Myocardial infarction (MI) & 377 & 9.9 & 71 & 6.7 & 120 & 5.0 & 568 & 7.8 \\
\hline Heart diseases (except MI) & 770 & 21.0 & 220 & 21.3 & 491 & 20.1 & $|48|$ & 21.0 \\
\hline Lung diseases & 261 & 7.0 & 80 & 7.7 & 169 & 7.1 & 510 & 7.1 \\
\hline Gastrointestinal diseases & 1298 & 34.6 & 322 & 30.6 & 657 & 27.6 & 2277 & 31.7 \\
\hline Pancreatic disease & 62 & 1.7 & 10 & 1.0 & 22 & 0.9 & 94 & 1.3 \\
\hline Liver/kidney diseases & 116 & 3.1 & 20 & 1.9 & 50 & 2.1 & 186 & 2.6 \\
\hline Muscle/bone diseases & 607 & 16.6 & 88 & 8.6 & 206 & 8.8 & 901 & 12.8 \\
\hline Central nervous system & 30 & 0.8 & 9 & 0.9 & 15 & 0.6 & 54 & 0.8 \\
\hline Cancer & 575 & 15.1 & 146 & 13.7 & 277 & 11.5 & 998 & 13.7 \\
\hline \multicolumn{9}{|l|}{ Women } \\
\hline Dyslipidemia & 1958 & 56.1 & 879 & 52.6 & 631 & 47.7 & 3468 & 53.5 \\
\hline Hyperuricemia & 234 & 6.8 & 70 & 4.3 & 50 & 3.8 & 354 & 5.6 \\
\hline Diabetes mellitus all & 390 & $\mathrm{II} .2$ & 112 & 6.7 & 55 & 4.2 & 557 & 8.6 \\
\hline insulin dependent & 80 & 2.3 & 24 & 1.4 & 10 & 0.8 & 114 & 1.8 \\
\hline Thyroid disease & 934 & 27.3 & 465 & 28.3 & 314 & 24.2 & 1713 & 30.0 \\
\hline Hypertension & 1916 & 54.9 & 799 & 47.8 & 544 & 41.2 & 3259 & 50.3 \\
\hline Stroke & 119 & 3.4 & 33 & 2.0 & 21 & 1.6 & 173 & 2.7 \\
\hline Myocardial infarction (MI) & 106 & 3.0 & 39 & 2.3 & 25 & 1.9 & 170 & 2.6 \\
\hline Heart diseases (except MI) & 560 & 16.8 & 286 & 17.8 & 212 & 16.5 & 1058 & 17.0 \\
\hline Lung diseases & 279 & 8.1 & 126 & 7.7 & 104 & 8.0 & 509 & 8.0 \\
\hline Gastrointestinal diseases & 1146 & 33.3 & 514 & 31.3 & 356 & 27.3 & 2016 & 31.6 \\
\hline Pancreatic diseases & 46 & 1.3 & 21 & 1.3 & 18 & $\mathrm{I} .4$ & 85 & 1.3 \\
\hline Liver/kidney diseases & 66 & 1.9 & 28 & 1.7 & 30 & 2.3 & 124 & 2.0 \\
\hline Muscle/bone diseases & 828 & 25.1 & 329 & 20.9 & 232 & 18.2 & 1389 & 22.6 \\
\hline Central nervous system & 20 & 0.6 & 8 & 0.5 & 7 & 0.5 & 35 & 0.5 \\
\hline Cancer & 452 & 12.9 & 220 & 13.2 & 179 & 13.5 & 851 & 13.1 \\
\hline
\end{tabular}

had little impact on the relationship between education and mortality. Further adjustment for potential confounders such as alcohol consumption, fruit and vegetable intake, and physical activity did not substantially influence the associations between education and multimorbidity in both men and women.

Table 5 shows the results of analyses stratified by age group. In participants aged $\leq 60$ years at recruitment, lower educational level was associated with higher prevalence of multimorbidity as well as for multiple metabolic diseases in men and in women. However, only in men the statistical interactions between age and education were significant for multimorbidity $(\mathrm{p}=0.008)$ and metabolic diseases $(\mathrm{p}=0.008)$ but not in women $(\mathrm{p}=0.808$ and $\mathrm{p}=$ 0.647).

\section{Discussion}

Overall, multimorbidity was more prevalent among men and women aged 50-75 years with low educational level than in subjects with higher educational level. Higher BMI, but not smoking status, was identified as an intermediate factor in the relationships between education and multimorbidity and metabolic disease. However, the adjustment for these risk factors did not completely explain the effect of education on health outcomes.

\section{Chronic diseases}

Consistent with our findings, other authors identified hypertension, dyslipidemia, chronic ischemic heart disease, back pain and diabetes as most prevalent diagnoses among 60 to 70 year old persons in Germany [23]. The higher prevalence of gastrointestinal diseases may be 
Table 2: Baseline characteristics by educational level and sex of participants aged 50 to 75 years in the EPIC-Heidelberg cohort

\begin{tabular}{|c|c|c|c|c|c|c|}
\hline & \multicolumn{3}{|c|}{ Men } & \multicolumn{3}{|c|}{ Women } \\
\hline $\begin{array}{l}\text { Educational } \\
\text { level }\end{array}$ & Low & Middle & High & Low & Middle & High \\
\hline $\mathbf{N}$ & 3810 & 1065 & 2418 & 3492 & 1670 & 1322 \\
\hline $\begin{array}{l}\text { Multimorbidity': } \\
\text { yes }(\%)\end{array}$ & 71.0 & 68.0 & 61.4 & 71.0 & 64.9 & 61.5 \\
\hline $\begin{array}{l}\text { Metabolic disease } 2: \\
\text { yes }(\%)\end{array}$ & 81.1 & 81.3 & 74.6 & 78.7 & 73.4 & 66.4 \\
\hline Deceased (\%) & 10.1 & 6.8 & 5.0 & 4.0 & 2.9 & 2.8 \\
\hline $\begin{array}{l}\text { Continuous } \\
\text { variables }\end{array}$ & Median (QI, Q3)* & Median (QI, Q3)* & Median (QI, Q3)* & Median (QI, Q3)* & Median (QI, Q3)* & Median (QI, Q3)* \\
\hline $\begin{array}{l}\text { Age at recruitment } \\
\text { (years) }\end{array}$ & $58(54,61)$ & $56(53,60)$ & $56(53,59)$ & $58(54,61)$ & $56(52,60)$ & $55(52,59)$ \\
\hline Height (m) & $\begin{array}{c}173.30 \\
(169.20,177.50)\end{array}$ & $\begin{array}{c}176.00 \\
(171.80,180.00)\end{array}$ & $\begin{array}{c}177.00 \\
(172.50,181.00)\end{array}$ & $\begin{array}{c}161.10 \\
(157.10,165.20)\end{array}$ & $\begin{array}{c}163.60 \\
(159.55,167.75)\end{array}$ & $\begin{array}{c}164.90 \\
(161.00,169.00)\end{array}$ \\
\hline Weight (kg) & $\begin{array}{c}83.30 \\
(83.30,91.40)\end{array}$ & $\begin{array}{c}82.50 \\
(75.70,90.50)\end{array}$ & $\begin{array}{c}81.20 \\
(75.50,90.00)\end{array}$ & $\begin{array}{c}70.00 \\
(63.00,79.05)\end{array}$ & $\begin{array}{c}67.25 \\
(60.50,75.60)\end{array}$ & $\begin{array}{c}65.00 \\
(59.00,72.00)\end{array}$ \\
\hline $\begin{array}{l}\text { Body mass index } \\
\left(\mathrm{kg} / \mathrm{m}^{2}\right)\end{array}$ & $27.6(25.6,30.0)$ & $26.7(24.7,29.1)$ & $25.9(24.1,28.1)$ & $26.9(24.3,30.3)$ & $25.1(22.7,28.0)$ & $23.9(21.8,26.6)$ \\
\hline $\begin{array}{l}\text { Total energy } \\
\text { intake }(\mathrm{kcal} / \mathrm{d})\end{array}$ & $2036(1678,2488)$ & $2006(1672,2432)$ & $2072(1725,2467)$ & $1603(1316,1935)$ & $1608(1350,1938)$ & $1677(1406,2000)$ \\
\hline Fat intake $(g / d)$ & $\begin{array}{c}74.10 \\
(57.60,95.97)\end{array}$ & $\begin{array}{c}74.67 \\
(58.37,93.68)\end{array}$ & $\begin{array}{c}75.06 \\
(59.66,95.02)\end{array}$ & $\begin{array}{c}61.77 \\
(48.64,77.96)\end{array}$ & $\begin{array}{c}61.81 \\
(48.79,76.42)\end{array}$ & $\begin{array}{c}62.49 \\
(50.48,78.44)\end{array}$ \\
\hline $\begin{array}{l}\text { Fruit consumption } \\
(\mathrm{g} / \mathrm{d})\end{array}$ & $\begin{array}{c}94.71 \\
(57.64,136.07)\end{array}$ & $\begin{array}{c}95.86 \\
(57.97,147.56)\end{array}$ & $\begin{array}{c}100.97 \\
(66.16 \quad 156.21)\end{array}$ & $\begin{array}{c}103.42 \\
(81.05,181.39)\end{array}$ & $\begin{array}{c}109.20 \\
(85.98,194.81)\end{array}$ & $\begin{array}{c}121.45 \\
(91.43,196.77)\end{array}$ \\
\hline $\begin{array}{l}\text { Vegetable } \\
\text { consumption }(g / d)\end{array}$ & $\begin{array}{c}99.27 \\
(74.74,131.09)\end{array}$ & $\begin{array}{c}103.02 \\
(78.33, \mid 31.53)\end{array}$ & $\begin{array}{c}108.72 \\
(84.14,140.74)\end{array}$ & $\begin{array}{c}106.10 \\
(81.29,143.60)\end{array}$ & $\begin{array}{c}110.02 \\
(85.67,146.44)\end{array}$ & $\begin{array}{c}122,11 \\
(93.19,161.12)\end{array}$ \\
\hline $\begin{array}{l}\text { Alcohol } \\
\text { consumption (g/d) }\end{array}$ & $18.13(6.93,39.77)$ & $19.99(7.80,39.16)$ & $20.93(8.89,38.97)$ & $4.04(1.06,11.39)$ & $6.89(2.18, \mid 7.31)$ & $9.05(2.80,21.82)$ \\
\hline $\begin{array}{l}\text { Categorical } \\
\text { variables }\end{array}$ & $\%$ & $\%$ & $\%$ & $\%$ & $\%$ & $\%$ \\
\hline \multicolumn{7}{|l|}{ Smoking status } \\
\hline Never smokers & 29.34 & 30.99 & 33.75 & 63.83 & 54.37 & 49.55 \\
\hline Former smokers & 48.35 & 47.79 & 52.23 & 20.36 & 29.46 & 35.33 \\
\hline Current Smokers & 22.31 & 21.22 & 14.02 & $15.8 \mid$ & 16.17 & 15.13 \\
\hline \multicolumn{7}{|l|}{$\begin{array}{l}\text { Combined total } \\
\text { physical activity } \\
\text { index }\end{array}$} \\
\hline Active & 8.50 & 3.10 & 2.70 & 9.68 & 5.95 & 7.34 \\
\hline Moderately active & 42.11 & 26.57 & 18.96 & 62.59 & 55.88 & 43.23 \\
\hline $\begin{array}{r}\text { Moderately } \\
\text { inactive }\end{array}$ & 33.94 & 30.97 & 29.69 & 21.96 & 25.42 & 29.70 \\
\hline Inactive & 15.44 & 39.36 & 48.65 & 5.76 & 12.75 & 19.72 \\
\hline
\end{tabular}

* Q I Cut point of Quartile I, Q3 Cut point of Quartile 3

IPresence of two or more of the he prevalent chronic conditions mentioned in table I

2Presence of hyperuricemia, diabetes mellitus, hypertension or dyslipidemia

attributed to younger age of the participants in our study. The prevalence of central-nervous system diseases, however, is likely to be underestimated because of the increasing study participants' disability to complete questionnaires.

Our observations are consistent with the well-known paradox that mortality in women is lower than in men of the same age despite higher levels of morbidity among women [24]. In our data, the sex difference of mortality was weaker compared to a previous review [25]. This may be explained by increasing smoking prevalence among women during the past decades [26].

\section{Intermediaries}

Differences in socio-economic position are associated with inequalities in health that may be mediated by differences in health-related behaviour [27]. In line with former 
Table 3: Age and multivariate adjusted odds ratios (OR, $95 \% \mathrm{CI})$ of the actual number of prevalent chronic diseases by educational level among men and women aged $\mathbf{5 0}$ to $\mathbf{7 5}$ years in the EPIC-Heidelberg cohort, applying multinomial logistic regression models (Reference category: highest educational level)

\begin{tabular}{|c|c|c|c|c|c|c|c|c|c|c|}
\hline \multirow{3}{*}{ Educational Level } & \multicolumn{5}{|c|}{ Men } & \multicolumn{5}{|c|}{ Women } \\
\hline & \multirow[b]{2}{*}{$\mathrm{N}$} & \multicolumn{2}{|c|}{ Middle } & \multicolumn{2}{|r|}{ Low } & \multirow[b]{2}{*}{$\mathrm{N}$} & \multicolumn{2}{|c|}{ Middle } & \multicolumn{2}{|r|}{ Low } \\
\hline & & OR & $95 \%-\mathrm{Cl}$ & OR & $95 \%-\mathrm{Cl}$ & & OR & $95 \%-\mathrm{Cl}$ & OR & $95 \%-\mathrm{Cl}$ \\
\hline \multicolumn{11}{|l|}{$\begin{array}{l}\text { Age-adjusted } \\
\text { Morbidity' }\end{array}$} \\
\hline 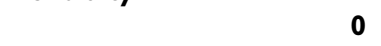 & 657 & 1 & & & & 576 & I & & & \\
\hline $\mathbf{I}$ & 1439 & 1.35 & $1.01-1.81$ & 1.08 & $0.89-1.32$ & 1249 & 1.31 & $1.00-1.71$ & 1.41 & $1.11-1.80$ \\
\hline 2 & 1595 & 1.51 & $1.14-2.02$ & 1.18 & $0.97-1.44$ & 1458 & 1.22 & $0.94-1.59$ & 1.50 & $1.19-1.91$ \\
\hline 3 & 1246 & 1.65 & $1.22-2.23$ & $\mathrm{I} .44$ & $1.16-1.77$ & 1124 & 1.29 & $0.98-|.7|$ & 1.64 & $1.28-2.11$ \\
\hline 4 & 800 & 1.69 & $1.21-2.36$ & 1.72 & $1.37-2.17$ & 634 & 1.67 & $|.22-2.3|$ & 1.85 & I.38-2.48 \\
\hline 5 & 374 & 2.00 & $1.30-3.09$ & 2.55 & $1.88-3.45$ & 335 & 1.77 & $1.16-2.68$ & 2.33 & $1.61-3.39$ \\
\hline$\geq 6$ & 293 & 1.96 & $1.19-3.23$ & 3.08 & $2.18-4.33$ & 218 & 1.54 & $0.89-2.66$ & 3.31 & $2.08-5.27$ \\
\hline$P$ for trend & & $<0.001$ & & 0.002 & & & 0.015 & & 0.003 & \\
\hline \multicolumn{11}{|l|}{ Multivariate-adjusted\# } \\
\hline \multicolumn{11}{|l|}{ Morbidity 1} \\
\hline 0 & 647 & 1 & & & & 566 & 1 & & & \\
\hline $\mathbf{I}$ & 1392 & 1.32 & $0.98-1.78$ & 1.02 & $0.82-1.27$ & 1182 & 1.26 & $0.96-1.66$ & 1.31 & $|.01-| .7 \mid$ \\
\hline 2 & 1511 & 1.44 & $1.07-1.93$ & 1.11 & $0.89-1.38$ & 1344 & 1.17 & $0.89-1.53$ & 1.30 & $1.00-1.68$ \\
\hline 3 & 1142 & 1.43 & $1.04-1.95$ & 1.19 & $0.94-1.49$ & 1000 & 1.11 & $0.82-1.48$ & 1.31 & $1.00-1.73$ \\
\hline 4 & 744 & 1.50 & $1.06-2.12$ & 1.39 & $1.07-1.79$ & 551 & 1.50 & $1.06-2.12$ & 1.30 & $0.94-1.81$ \\
\hline 5 & 353 & 1.64 & $1.04-2.57$ & 1.76 & $1.26-2.45$ & 285 & 1.47 & $0.94-2.32$ & 1.60 & $1.05-2.43$ \\
\hline$\geq 6$ & 253 & 1.44 & $0.83-2.49$ & 2.24 & $1.52-3.29$ & 186 & 1.47 & $0.78-2.76$ & 2.53 & $\mid .45-4.4 \mathrm{I}$ \\
\hline$P$ for trend & & 0.039 & & 0.003 & & & 0.021 & & 0.039 & \\
\hline \multirow{2}{*}{\multicolumn{11}{|c|}{$\begin{array}{l}\text { Age-adjusted } \\
\text { Metabolic diseases }^{2}\end{array}$}} \\
\hline & & & & & & & & & & \\
\hline 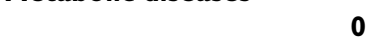 & 1504 & 1 & & & & 1606 & I & & & \\
\hline $\mathbf{I}$ & 2480 & 1.36 & $1.11-1.66$ & 1.10 & $0.96-1.27$ & 2562 & 1.28 & $1.08-1.53$ & 1.39 & $1.19-1.63$ \\
\hline 2 & 2121 & 1.53 & $1.24-1.88$ & 1.41 & $1.22-1.63$ & 1749 & 1.43 & $1.17-1.75$ & 1.85 & $1.55-2.22$ \\
\hline $3 / 4$ & 1054 & 1.81 & $1.39-2.36$ & 2.40 & $1.99-2.89$ & 454 & 1.72 & $1.18-2.50$ & 3.08 & $2.23-4.26$ \\
\hline$P$ for trend & & 0.009 & & 0.089 & & & 0.007 & & 0.042 & \\
\hline \multicolumn{11}{|l|}{$\begin{array}{l}\text { Multivariate-adjusted } \# \\
\text { Metabolic diseases }^{2}\end{array}$} \\
\hline 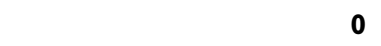 & 1422 & I & & & & 1483 & 1 & & & \\
\hline $\mathbf{I}$ & 2334 & 1.30 & $1.06-1.61$ & 0.99 & $0.85-1.16$ & 2329 & 1.19 & $0.99-1.43$ & 1.24 & $1.04-1.48$ \\
\hline 2 & 2004 & 1.36 & $1.09-1.69$ & 1.16 & $0.98-1.37$ & 1602 & 1.29 & $1.04-1.60$ & 1.38 & $1.13-1.69$ \\
\hline $3 / 4$ & 999 & 1.51 & $1.14-2.02$ & 1.62 & $1.31-2.00$ & 402 & 1.40 & $0.93-2.12$ & 1.76 & $1.21-2.55$ \\
\hline $\mathrm{P}$ for trend & & 0.043 & & 0.113 & & & 0.012 & & 0.018 & \\
\hline
\end{tabular}

\# adjusted for smoking status, BMI (continuous; kg/m2), fruit intake (continuous; g/day), vegetable intake (continuous; g/day), alcohol consumption (continuous; g/day), age (continuous; years), combined total physical activity index (inactive, moderately inactive, moderately active, active)

'Number of the prevalent chronic conditions mentioned in table I

${ }^{2}$ Number of prevalent metabolic diseases, including hyperuricemia, diabetes mellitus, hypertension and dyslipidemia

research [15,27-30], we observed that participants with a higher educational level were leaner, ate more fruits and vegetables, and were less likely to be smokers at recruitment. Consistent with our results, it was also noted that moderate drinking was not associated with a SEP gradient, while heavy drinking was more prevalent at lower levels $[31,32]$. We observed that the smoking prevalence and, in consequence, number of smoking related diseases were higher in low education groups than in their counterparts. This observation is in consistency with other reports $[28,31]$. In our study, however, middle and highly educated men were less physically active than less educated men (please see table 2). The combination of physical inactivity and other adverse lifestyle factors could explain the high prevalence of metabolic disease in men at intermediate educational level.

Our observation that low SEP was recognized as a determinant for higher risk of chronic diseases are in line with the literature $[5,6,31]$. We observed that the association of education differed by disease and adjustment attenuated the relationships differentially $[6,33]$. Our results suggest that higher BMI, but not smoking status, may act as an intermediate factor of education on multimorbidity. However, smoking status attenuated the estimates for mortality. Potential explanation could be that in the age 
Table 4: Age and multivariate adjusted odds (OR, $95 \% \mathrm{Cl}$ ) and hazard ratios (HR, $95 \% \mathrm{Cl}$ ) of multimorbidity, metabolic diseases, and mortality, including additional models adjusted for smoking status or BMI, by educational level among men and women aged $\mathbf{5 0}$ to $\mathbf{7 5}$ years in the EPIC-Heidelberg cohort

\begin{tabular}{|c|c|c|c|c|c|c|c|c|c|c|c|}
\hline \multirow{3}{*}{ Educational level } & \multicolumn{6}{|c|}{ Men } & \multicolumn{5}{|c|}{ Women } \\
\hline & \multicolumn{2}{|l|}{ High } & \multicolumn{2}{|c|}{ Middle } & \multicolumn{2}{|r|}{ Low } & \multirow[t]{2}{*}{ High } & \multicolumn{2}{|c|}{ Middle } & \multicolumn{2}{|c|}{ Low } \\
\hline & OR & & OR & $95 \%-\mathrm{Cl}$ & OR & $95 \%-\mathrm{Cl}$ & & OR & $95 \%-\mathrm{Cl}$ & OR & $95 \%-\mathrm{Cl}$ \\
\hline \multicolumn{12}{|l|}{ Multimorbidity: yes' } \\
\hline Age-adjusted & & 1 & 1.32 & $1.12-1.55$ & 1.43 & $|.28-1.6|$ & 1 & 1.13 & $0.97-1.33$ & 1.33 & $1.18-1.57$ \\
\hline Smoking status -adjusted $*$ & & 1 & 1.34 & I.14-1.58 & 1.46 & $1.30-1.64$ & 1 & 1.14 & $0.97-1.34$ & 1.38 & $1.20-1.60$ \\
\hline BMI- adjusted $* *$ & & 1 & 1.23 & $1.05-1.45$ & 1.24 & $1.10-1.40$ & 1 & 1.05 & $0.89-1.23$ & 1.12 & $0.96-1.30$ \\
\hline Fully adjusted \# & & I & 1.21 & $1.02-1.43$ & 1.28 & $1.12-1.46$ & I & 1.06 & $0.89-1.25$ & 1.16 & $0.99-1.36$ \\
\hline \multicolumn{12}{|l|}{ Metabolic diseases: yes ${ }^{2}$} \\
\hline Age-adjusted & & 1 & 1.48 & $1.23-1.77$ & 1.38 & $1.22-1.57$ & 1 & 1.35 & $1.15-1.59$ & 1.64 & $1.42-1.90$ \\
\hline Smoking status -adjusted $*$ & & i & 1.51 & $1.26-1.81$ & 1.41 & $1.25-1.61$ & I & 1.36 & $1.16-1.60$ & 1.67 & $1.44-1.93$ \\
\hline BMI- adjusted $* *$ & & 1 & 1.34 & $1.11-1.61$ & 1.09 & $0.96-1.25$ & 1 & 1.21 & $1.03-1.42$ & 1.25 & $1.18-1.46$ \\
\hline Fully adjusted \# & & I & 1.37 & $1.13-1.66$ & 1.15 & $1.00-1.33$ & I & 1.24 & $1.04-1.47$ & 1.33 & $1.13-1.56$ \\
\hline & $H R$ & & $H R$ & $95 \%-\mathrm{Cl}$ & HR & $95 \%-\mathrm{Cl}$ & & $H R$ & $95 \%-\mathrm{Cl}$ & $\mathrm{HR}$ & $95 \%-\mathrm{Cl}$ \\
\hline \multicolumn{12}{|l|}{ All-cause mortality: yes } \\
\hline Age-adjusted & & I & 1.38 & $1.02-1.85$ & 1.90 & $\mathrm{I} .54-2.34$ & I & 1.04 & $0.67-1.61$ & 1.30 & $0.89-1.90$ \\
\hline Smoking status -adjusted $*$ & & I & 1.26 & $0.94-1.69$ & 1.69 & $1.37-2.09$ & I & 1.05 & $0.68-1.62$ & 1.33 & $0.92-1.95$ \\
\hline BMI- adjusted $* *$ & & 1 & 1.36 & $1.01-1.83$ & 1.82 & $1.46-2.25$ & 1 & 0.99 & $0.64-1.54$ & 1.23 & $0.83-1.81$ \\
\hline Fully adjusted \# & & 1 & 1.26 & $0.91-1.74$ & 1.60 & $1.25-2.05$ & 1 & 1.32 & $0.78-2.22$ & 1.49 & $0.92-2.41$ \\
\hline
\end{tabular}

* adjusted for smoking status and age (continuous; years)

** adjusted for BMI (continuous; $\mathrm{kg} / \mathrm{m}^{2}$ ), and age (continuous; years)

\# adjusted for smoking status, BMI (continuous; $\mathrm{kg} / \mathrm{m}^{2}$ ), fruit intake (continuous; $\mathrm{g} /$ day), vegetable intake (continuous; $\mathrm{g} / \mathrm{day}$ ), alcohol consumption

(continuous; g/day), age (continuous; years), combined total physical activity index (inactive, moderately inactive, moderately active, active)

'Presence of two or more of the prevalent chronic conditions mentioned in table I

2Presence of prevalent metabolic diseases, including hyperuricemia, diabetes mellitus, hypertension or dyslipidemia

group 50 to 75 years smoking habits are rather associated with mortality than with multimorbidity.

Research on mortality has shown that the associations with education were partially eliminated after adjustment for covariates [34]. Other authors found that both social class in childhood and adulthood contribute to disease risk applying objective measures in mid-life [35].

\section{Multimorbidity}

Consistent with other studies, we observed that low educational level was statistically significantly associated with a higher prevalence of multimorbidity compared to high

Table 5: Multivariate adjusted odds ratios $(\mathrm{OR}, 95 \% \mathrm{CI})$ of multimorbidity and metabolic diseases by educational level and sex according to age groups among participants aged 50 to 75 years in the EPIC-Heidelberg cohort

\begin{tabular}{|c|c|c|c|c|c|c|c|c|c|c|}
\hline \multirow{3}{*}{ Educational Level } & \multicolumn{5}{|c|}{ Men } & \multicolumn{5}{|c|}{ Women } \\
\hline & High & Middle & & Low & & High & Middle & & Low & \\
\hline & OR & OR & $95 \%-\mathrm{Cl}$ & OR & $95 \%-\mathrm{Cl}$ & OR & OR & $95 \%-\mathrm{Cl}$ & OR & $95 \%-\mathrm{Cl}$ \\
\hline \multicolumn{11}{|l|}{ Multimorbidity: yes' } \\
\hline Age $<=60$ years at recruitment $\#$ & 1 & 1.24 & $1.03-1.50$ & 1.36 & $1.17-1.59$ & I & 1.05 & $0.87-1.26$ & 1.20 & $1.00-1.43$ \\
\hline Age $>60$ years at recruitment \# & 1 & 0.95 & $0.65-1.39$ & 0.90 & $0.68-1.19$ & I & 1.08 & $0.70-1.67$ & 0.93 & $0.64-1.35$ \\
\hline \multicolumn{11}{|l|}{ Metabolic diseases: yes ${ }^{2}$} \\
\hline Age $<=60$ years at recruitment \# & 1 & 1.38 & $1 .|2-1.7|$ & 1.18 & $1.00-1.38$ & 1 & 1.24 & $1.03-1.49$ & 1.32 & $1.11-1.58$ \\
\hline Age $>60$ years at recruitment $\#$ & 1 & 1.16 & $0.74-1.83$ & 0.97 & $0.71-1.32$ & 1 & 1.16 & $0.74-1.82$ & 1.24 & $0.84-1.83$ \\
\hline
\end{tabular}

$\S$ adjusted for BMI (continuous; $\mathrm{kg} / \mathrm{m} 2$ ), fruit intake (continuous; g/day), vegetable intake (continuous, g/day), alcohol consumption (continuous; g/ day), age (continuous; years, total physical activity (inactive, moderately inactive, moderately active, active)

* adjusted for smoking status, fruit intake (continuous; g/day), vegetable intake (continuous; g/day), alcohol consumption (continuous; g/day), age (continuous years), combined total physical activity index (inactive, moderately inactive, moderately active, active)

\# adjusted for smoking status, BMI (continuous; $\mathrm{kg} / \mathrm{m} 2$ ), fruit intake (continuous; g/day), vegetable intake (continuous; g/day), alcohol consumption (continuous; g/day), age (continuous years), combined total physical activity index (inactive, moderately inactive, moderately active, active) 'Presence of two or more of the prevalent chronic conditions mentioned in table I

2Presence of hyperuricemia, diabetes mellitus, hypertension or dyslipidemia 
educational level $[2,6]$. Stronger associations between education and multimorbidity were found among the participants aged 50 to 60 years than among elders, which can be explained by premature mortality in low SEP groups [36]. Growing evidence exists that SEP differences are also reflected in biological markers of disease [5,6]. In a cohort study on cardiovascular risk factors among men in France and Northern Ireland, education was inversely associated with blood pressure and BMI but not with total cholesterol concentration [31]. Our data further suggest that the educational attainment is not only associated with multimorbidity, but that the social gradient is related to the number of diseases. Our observation that education has an impact on multimorbidity is supported by numerous studies on the relationship between low education and high mortality $[9,13,23]$. Further evidence comes from a study on morbidity during the last years of life among US citizens, which revealed lower morbidity and disability in those with higher education [37].

Further unmeasured factors may contribute to the relationship between education and multimorbidity such as psychosocial factors, stress and other environmental factors $[7,38-40]$. It has been hypothesized that low control in terms of autonomy and low social participation are likely contributors to health inequalities [41]. Psychosocial variables such as employment, active coping style, high occupational class and an external locus of control were associated with low prevalence of multimorbidity [42].

Sex differences in health were hypothesized to be related to differential exposure and differential vulnerability [43]. In the present study, pancreatitis and hyperuricemia were more frequent in men and stroke was more frequent in women. Higher prevalence of diabetes in women than in men was found by other authors $[6,44]$. Our results suggest differential associations between education and multimorbidity by sex, which may be explained by differences in lifestyle [26], however, these differences could also be due to chance variation by stratification. Also behavioural aspects are likely to be linked with the outcome $[40,43]$.

Some potential limitations of this study should be considered. We analyzed school attainment as indicator of SEP, but no other indicator such as profession or income. However, measures for adulthood SEP such as occupation or income and measures of economic distress have been shown to be in good correlation with each other [45]. Participants of EPIC-Heidelberg were recruited from the general population [19], but the response rate in less educated subjects tends to be lower than in well-educated ones, implying that the real differences may actually be underestimated.
Our study was largely based on the participants' selfreports, which could have introduced some bias. However, all self-reports on cancer were verified by comparison with medical records, and validation studies were conducted for diabetes, myocardial infarction, and stroke. Misreporting could be linked to the respondents' educational levels, resulting in underestimation of educational inequalities in a previous study [46]. Also, non-response is higher in groups of low SEP and of ill-health [47], which may also result in attenuated estimates. Information on habitual diet and lifestyle factors may be affected by morbidity itself since reverse causation can not be excluded. However, dietary habits and other lifestyle factors were found to be relatively stable over time in our cohort [48]. For some chronic diseases it is difficult to determine whether or not they can be considered independently from each other, such as diabetes mellitus, hypertension, and myocardial infarction [49]. From the public health point-of-view, however, this is irrelevant because persons with several diseases are likely to take more medications and to have more medical consultations.

The strengths of this study are the use of prospective data, its population-based approach, and the possibility to adjust for relevant confounding variables.

\section{Conclusion}

In this large prospective study, low educational attainment was associated with higher prevalence of multimorbidity. Our results suggest that higher BMI is an intermediate factor for multimorbidity among subjects aged 50 to 75 years. Adjustment for other potential risk factors did not completely explain the relationships. Aspects of SEP, sex and BMI need consideration in public health actions to prevent multimorbidity.

\section{Competing interests}

The authors declare that they have no competing interests.

\section{Authors' contributions}

GN and RP initiated and designed the project and GN conducted the statistical analyses. JL is principal investigator of the EPIC-Heidelberg cohort study and contributed to the study design. SR and SH were involved in data collection and contributed to the variable selection. SB and SR provided advice on design and statistics. All authors provided specialist knowledge in the advancement of the analyses and provided input in re-drafting the manuscript. GN drafted the first manuscript and wrote the final version, which was seen and approved by all authors.

\section{Acknowledgements}

We thank all EPIC-Heidelberg participants as well as Dorothee Zoller and Jutta Schmitt for their excellent support in data management. 
Funding: The EPIC-Heidelberg study was funded by "Europe Against Cancer" Programme of the European Commission (SANCO); German Cancer Aid; German Cancer Research Center; German Federal Ministry of Education and Research, and Kurt-Eberhard-Bode-Stiftung.

\section{References}

I. Feinstein AR: The pretherapeutic classification of chronic disease. J Chron Dis 1979, 23:455-68.

2. Akker M van den, Buntinx F, Metsemakers JF, Roos S, Knottnerus JA Multimorbidity in general practice: prevalence, incidence, and determinants of co-occurring chronic and recurrent diseases. I Clin Epidemiol 1998, 5 I:367-75.

3. Fortin M, Bravo G, Hudon C, Vanasse A, Lapointe L: Prevalence of multimorbidity among adults seen in family practice. Ann Fam Med 2005, 3:223-8.

4. Wolff JL, Starfield B, Anderson G: Prevalence, expenditures, and complications of multiple chronic conditions in the elderly. Arch Intern Med 2002, 162:2269-76.

5. Banks J, Marmot M, Oldfield Z, Smith JP: Disease and disadvantage in the United States and in England. JAMA 2006, 295:2037-45.

6. Dalstra JA, Kunst AE, Borrell C, Breeze E, Cambois E, Costa G, et al. Socioeconomic differences in the prevalence of common chronic diseases: an overview of eight European countries. Int J Epidemiol 2005, 34:316-26.

7. Peter R, Gassler H, Geyer S: Socioeconomic status, status inconsistency and risk of ischaemic heart disease: a prospective study among members of a statutory health insurance company. J Epidemiol Community Health 2007, 6 I:605-I I.

8. Wong MD, Shapiro MF, Boscardin W], Ettner SL: Contribution of major diseases to disparities in mortality. N Engl J Med 2002 347: I585-92.

9. Huisman M, Kunst AE, Bopp M, Borgan JK, Borrell C, Costa G, et al.: Educational inequalities in cause-specific mortality in middle-aged and older men and women in eight western European populations. Lancet 2005, 365:493-500.

10. Isaacs SL, Schroeder SA: Class - the ignored determinant of the nation's health. N Engl ] Med 2004, 35 I: I I37-42

II. Nagel G, Linseisen J, Boshuizen HC, Pera G, Del Giudice G, Westert GP, et al.: Socioeconomic position and the risk of gastric and oesophageal cancer in the European Prospective Investigation into Cancer and Nutrition (EPIC-EURGAST). Int J Epidemiol 2007, 36:66-76.

12. Krieger N, Williams DR, Moss NE: Measuring social class in US public health research: concepts, methodologies, and guidelines. Annu Rev Public Health 1997, 1 8:341-78.

13. Adler NE, Newman K: Socioeconomic disparities in health: pathways and policies. Health Aff (Millwood) 2002, 21:60-76.

14. Huisman M, Kunst AE, Mackenbach JP: Inequalities in the prevalence of smoking in the European Union: comparing education and income. Prev Med 2005, 40:756-64.

15. Smith GD, Brunner E: Socio-economic differentials in health: the role of nutrition. Proc Nutr Soc 1997, 56:75-90.

16. Lantz PM, House JS, Lepkowski JM, Williams DR, Mero RP, Chen J: Socioeconomic factors, health behaviors, and mortality: results from a nationally representative prospective study of US adults. JAMA 1998, 279:1703-8.

17. Fortin M, Soubhi H, Hudon C, Bayliss EA, Akker M van den: Multimorbidity's many challenges. BMJ 2007, 334:1016-7.

18. Riboli E, Kaaks R: The EPIC Project: rationale and study design. European Prospective Investigation into Cancer and Nutrition. Int J Epidemiol I997, 26(Suppl I):S6-14.

19. Boeing H, Korfmann A, Bergmann MM: Recruitment procedures of EPIC-Germany. European Investigation into Cancer and Nutrition. Ann Nutr Metab 1999, 43:205-15.

20. Nagel G, Linseisen J: Dietary intake of fatty acids, antioxidants and selected food groups and asthma in adults. Eur J Clin Nutr 2005, 59:8- 15

21. Wareham NJ, Jakes RW, Rennie KL, Schuit J, Mitchell J, Hennings S, Day NE: Validity and repeatability of a simple index derived from the short physical activity questionnaire used in the European Prospective Investigation into Cancer and Nutrition (EPIC) study. Public Health Nutr 2003, 6:407-I3.

22. Denton M, Prus S, Walters V: Gender differences in health: Canadian study of the psychosocial, structural and behavioural determinants of health. Soc Sci Med 2004, 58:2585-600.
23. Walter U, Schneider N, Bisson S: [Morbidity and health in old age. A challenge for prevention and health care]. Bundesgesundheitsblatt Gesundheitsforschung Gesundheitsschutz 2006, 49:537-46.

24. Verbrugge LM: The twain meet: empirical explanations of sex differences in health and mortality. J Health Soc Behav 1989, 30:282-304

25. Mustard CA, Etches ]: Gender differences in socioeconomic inequality in mortality. J Epidemiol Community Health 2003, 57:974-80.

26. Rohrmann S, Kroke A, Boeing $\mathrm{H}$, Becker $\mathrm{N}$ : Time trends in cigarette smoking in two German cohorts-results from EPIC Germany. Eur J Cancer Prev 2003, 1 2:327-32.

27. Shahar D, Shai I, Vardi H, Shahar A, Fraser D: Diet and eating habits in high and low socioeconomic groups. Nutrition 2005 , 21:559-66.

28. Giskes K, Kunst AE, Benach J, Borrell C, Costa G, Dahl E, et al: Trends in smoking behaviour between 1985 and 2000 in nine European countries by education. J Epidemiol Community Health 2005, 59:395-40I.

29. Giskes K, Lenthe FF, Brug HJ, Mackenbach J: Dietary intakes of adults in the Netherlands by childhood and adulthood socioeconomic position. Eur J Clin Nutr 2004, 58:87I-80.

30. Irala-Estevez JD, Groth M, Johansson L, Oltersdorf U, Prattala R, Martinez-Gonzalez MA: A systematic review of socio-economic differences in food habits in Europe: consumption of fruit and vegetables. Eur J Clin Nutr 2000, 54:706-I4.

31. Yarnell J, Yu S, McCrum E, Arveiler D, Hass B, Dallongeville J, et al:: Education, socioeconomic and lifestyle factors, and risk of coronary heart disease: the PRIME Study. Int J Epidemiol 2005, 34:268-75.

32. Murray RP, Connett JE, Tyas SL, Bond R, Ekuma O, Silversides CK, Barnes GE: Alcohol volume, drinking pattern, and cardiovascular disease morbidity and mortality: is there a U-shaped function? Am J Epidemiol 2002, 155:242-8.

33. Power C, Matthews S: Origins of health inequalities in a national population sample. Lancet 1997, 350:1584-9.

34. Kilander L, Berglund L, Boberg M, Vessby B, Lithell H: Education, lifestyle factors and mortality from cardiovascular disease and cancer. A 25-year follow-up of Swedish 50-year-old men. Int J Epidemiol 2001, 30: I I 19-26.

35. Power C, Atherton K, Strachan DP, Shepherd P, Fuller E, Davis A, et al.: Life-course influences on health in British adults: effects of socio-economic position in childhood and adulthood. Int J Epidemiol 2007

36. Arber S, Ginn J: Gender and inequalities in health in later life. Soc Sci Med 1993, 36:33-46.

37. Liao Y, McGee DL, Kaufman JS, Cao G, Cooper RS: Socioeconomic status and morbidity in the last years of life. Am J Public Health 1999, 89:569-72.

38. Forastiere F, Stafoggia M, Tasco C, Picciotto S, Agabiti N, Cesaroni G, Perucci CA: Socioeconomic status, particulate air pollution, and daily mortality: differential exposure or differential susceptibility. Am J Ind Med 2007, 50:208-16.

39. Steptoe A, Kunz-Ebrecht S, Owen N, Feldman PJ, Willemsen G, Kirschbaum C, Marmot M: Socioeconomic status and stressrelated biological responses over the working day. Psychosom Med 2003, 65:46I-70.

40. Christakis NA, Fowler JH: The spread of obesity in a large social network over 32 years. N Engl J Med 2007, 357:370-9.

41. Marmot MG: Status syndrome: a challenge to medicine. JAMA 2006, 295: I304-7.

42. Akker M van den, Buntinx F, Metsemakers JF, Knottnerus JA: Marginal impact of psychosocial factors on multimorbidity: results of an explorative nested case-control study. Soc Sci Med 2000, 50:1679-93.

43. Macintyre S, Hunt $\mathrm{K}$, Sweeting $\mathrm{H}$ : Gender differences in health: are things really as simple as they seem? Soc Sci Med 1996, 42:617-24.

44. Agardh EE, Ahlbom A, Andersson T, Efendic S, Grill V, Hallqvist J, Ostenson CG: Socio-economic position at three points in life in association with type $\mathbf{2}$ diabetes and impaired glucose tolerance in middle-aged Swedish men and women. Int J Epidemiol 2007, 36:84-92.

45. Lahelma E, Martikainen P, Laaksonen M, Aittomaki A: Pathways between socioeconomic determinants of health. J Epidemio Community Health 2004, 58:327-32. 
46. Mackenbach JP, Looman CW, Meer JB van der: Differences in the misreporting of chronic conditions, by level of education: the effect on inequalities in prevalence rates. Am J Public Health 1996, 86:706-II.

47. Dengler $R$, Roberts $H$, Rushton $L$ : Lifestyle surveys-the complete answer? J Epidemiol Community Health 1997, 5 I:46-5I.

48. Nagel G, Zoller D, Ruf T, Rohrmann S, Linseisen J: Long-term reproducibility of a food-frequency questionnaire and dietary changes in the European Prospective Investigation into Cancer and Nutrition (EPIC)-Heidelberg cohort. Br J Nutr 2007, 98:194-200.

49. Akker $M$ van den, Buntinx F, Roos S, Knottnerus JA: Problems in determining occurrence rates of multimorbidity. J Clin Epidemiol 200I, 54:675-9.

\section{Pre-publication history}

The pre-publication history for this paper can be accessed here:

http://www.biomedcentral.com/1471-2458/8/384/pre

pub

Publish with Bio Med Central and every scientist can read your work free of charge

"BioMed Central will be the most significant development for disseminating the results of biomedical research in our lifetime. "

Sir Paul Nurse, Cancer Research UK

Your research papers will be:

- available free of charge to the entire biomedical community

- peer reviewed and published immediately upon acceptance

- cited in PubMed and archived on PubMed Central

- yours - you keep the copyright

Submit your manuscript here:

http://www.biomedcentral.com/info/publishing_adv.asp
BioMedcentral 